\title{
Comparative analysis of the SpinoMeter mobile application and Surgimap system for measuring the sagittal balance parameters: inter-observer reliability test
}

\author{
D.V. Ivanov ${ }^{1}$, I.V. Kirillova ${ }^{1}$, L.Yu. Kossovich ${ }^{1}$, S.V. Likhachev ${ }^{2}$, A.V. Polienko ${ }^{1}$, A.V. Kharlamov ${ }^{1}$, \\ A.E. Shulga ${ }^{2}$
}

${ }^{1}$ Saratov State University, Saratov, Russian Federation

${ }^{2}$ Saratov State Medical University named after V.I. Razumovsky, Saratov, Russian Federation

\begin{abstract}
The purpose of the study was to assess the reliability, accuracy and effectiveness of a mobile application SpinoMeter for measuring the sagittal balance of the spino-pelvic complex in comparison with the desktop preoperative planning system Surgimap. Methods SpinoMeter mobile application was developed in 2019 at the laboratory for Medical Decision Support Systems of Saratov State University. SpinoMeter is intended for measuring the actual and for calculating the optimal parameters of the sagittal balance of the spino-pelvic complex of a particular patient. Pelvic incidence (PI), lumbar lordosis (LL), sacral slope (SS) and pelvic tilt (PT) were measured with the help of Surgimap and SpinoMeter. Inter-observer reliability was assessed on X-rays in a standing position of 52 patients having consequences of injuries and pathology of the lumbar spine with imbalance syndrome. The research site was the Scientific Research Institute of Traumatology, Orthopedics and Neurosurgery of Saratov State Medical University. Measurements of the sagittal balance parameters were presented in tables and diagrams. For all data, the hypothesis of a normal distribution of the measurement results was tested using the Kolmogorov-Smirnov test. To test the hypothesis of homogeneity of the measurement results for normally distributed samples, the paired Student $t$-test was used. In the absence of a normal distribution, the Wilcoxon test was used for related samples. Results For PI, PT, SS and L1-S1 lordosis at a significance level of $5 \%$, the hypothesis of uniformity of measurements was confirmed. The average value of L4-S1 lordosis, measured by SpinoMeter, was shifted down relative to the measurements of this parameter by the Surgimap program. The shift did not exceed $0.7 \%$. Conclusion SpinoMeter mobile application is equivalent to the Surgimap system in terms of measuring the sagittal balance parameters based on radiographs in a standing position.
\end{abstract}

Keywords: sagittal balance, mobile application, sagittal balance parameters

\section{INTRODUCTION}

The term "spino-pelvic balance" was first introduced by Duval-Beaupere [1]. Today, such wellknown foreign and domestic scientists and clinicians as Le Huec [2], Shwabb [3], Krutko [4] and others devote their fundamental and applied studies to the sagittal balance of the spino-pelvic complex.

It is believed [5] that maintaining the sagittal balance or neutral vertical alignment of the spine in the sagittal plane is the main goal of surgical, ergonomic and physiotherapy procedures. The optimal sagittal balance allows for an optimal stress-strain state of the spine and implants in terms of biomechanics. Moreover, the need to correct the sagittal profile of a patient by performing operations on the spino-pelvic complex was substantiated from the point of view of biomechanics [6]. Modern studies show [7, 8] that the sagittal balance plays an important role in the development of degenerative changes in the adjacent spinal motion segments (SMS) in extended fixation. It was revealed that patients with post-operative sagittal imbalance had significantly higher risks of degenerative changes in adjacent SMS [7].
Sagittal balance parameters have been used $[9,10]$ to assess the postoperative period and complications in the treatment of patients with degenerative diseases of the spine and pelvic complex (SPC).

New tools for assessing sagittal balance parameters are available to clinicians and researchers: PACS systems [11], preoperative planning systems [12] for desktop computers, and mobile applications for smartphones $[13,14]$. The latter are, in our opinion, an extremely promising tool for practicing surgeons and radiologists.

Our work is a comparative assessment of the accuracy and reproducibility of measurements of the sagittal balance parameters performed using the SpinoMeter mobile application. The Surgimap system, which has been used by domestic [15] and foreign [16] doctors for planning surgical treatment in spinal pathology involving the spinal-pelvic junction, was considered as a "standard".

The angular parameters of the sagittal balance were measured: PI, PT, SS, lordosis at the L4-S1 level and lordosis at the L1-S1 level, which are shown in Figure 1. 

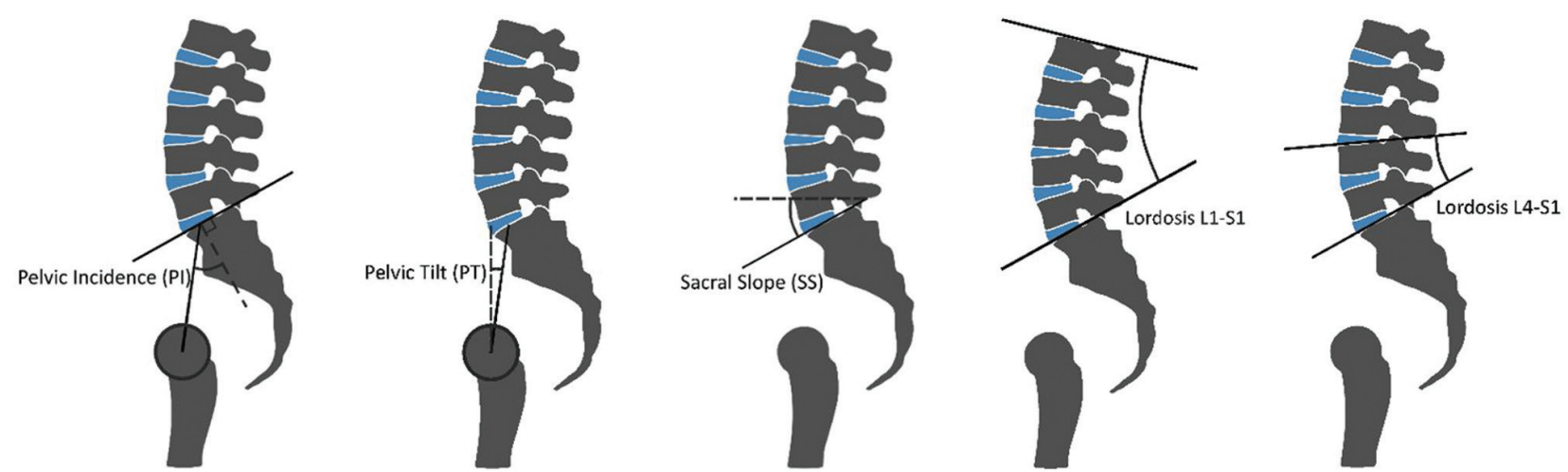

Fig. 1 Measured parameters of the sagittal balance (from left to right): PI, PT, SS lordosis at L1-S1 level, lordosis at L4-S1 level

\section{MATERIAL AND METHODS}

The material for the study was the lateral radiographs of the spine and pelvis performed in the patients of SMSU. Inclusion criteria were the radiographs of the quality sufficient to determine the boundaries of the vertebrae and femoral heads, visualization of the L1S1 vertebrae and femoral heads on one radiograph, and radiographs in the standing position. The average age of the patients was 46 years (range, 32-57 years). The study included radiographs of 52 patients. There were 24 (46\% of the total) patients with spinal trauma, and 28 (54\% of the total) patients with Meyerding spondylolisthesis in grade 3-4.

We used two software tools for measuring the parameters of the sagittal balance in radiographs: the Surgimap desktop application version 2.3.1.2 (115 East 23rd St, Suite \# 501, New York, NY 10010, USA) and the SpinoMeter mobile application (certificate of state computer program registration, registration number 2019665169 from 20.11.2019).

Detailed information about the Surgimap program is posted on the manufacturer's website [12]. The SpinoMeter mobile application was developed during the implementation of the project "Development of a prototype of a medical decision support system in reconstructive surgery of the spine and pelvic complex" of the Foundation for Advanced Research. The development and support of the application is carried out by the laboratory "Systems of support for medical decision making" at N.G. Chernyshevsky Saratov State University.

The SpinoMeter mobile application provides measurements of the actual parameters of the sagittal balance of the spine in radiographs, on a computer monitor for a specific patient and calculation of their theoretical (optimal) values. During the operation of the application, only the sensors of the smartphone position in space are used, which allows working at any room light and quality of radiographs.

First, we provide the main parameters of the sagittal balance which are measured and calculated by the SpinoMeter.
Pelvic parameters:

- Pelvic Incidence (PI) - angle between the line drawn from the center of the $\mathrm{S} 1$ endplate to the femoral head and the line perpendicular to S1 endplate;

- Pelvic Tilt (PT) - angle between the line drawn from the center of S1 endplate to the femoral head, and the vertical line;

- Sacral Slope (SS) - angle between the horizontal line and $\mathrm{S} 1$ endplate.

Spine parameters:

- Lordosis L4-S1 - angle between S1 endplate and upper L4 endplate;

- Global Lordosis L1-S1 (GLL) - angle between L1 and S1 endplates.

Additional parameters of the sagittal balance can be measured with SpinoMeter.

- Spino-Cranial Angle (SCA) - angle between the line drawn through the $\mathrm{C} 7$ lower endplate and the line connecting the sella turcica center and the support point of $\mathrm{C} 7$;

- Spino-Sacral Angle (SSA) - angle between the $\mathrm{S} 1$ endplate and the line connecting the center of C7 with the middle of the $\mathrm{S} 1$ endplate;

- Lordosis C2-C7 - angle between the C7 lower endplate and $\mathrm{C} 2$ lower endplate;

- Occipito-C2 Angle - angle between the C2 endplate and MacGregor line;

- C7 Slope - angle between the lower endplate of $\mathrm{C} 7$ and horizontal line.

Full Balance Integrated index (FBI) also may be calculated with this mobile application. Three angles are assessed for its calculation:

- C7TA - angle of translation C7;

- Angle of femur obliquity (FOA) - angle of femur inclination relative to the vertical line;

- Angle of pelvic tilt compensation (PTCA) angle of compensatory pelvic tilt.

Then the FBI is calculated according to the formula FBI $=$ C7TA + FOA + PTCA.

The results of measurements are accumulated in 
the mobile phone and may be sent to colleagues with the application (mail, SMS, messenger). All main parameters of the sagittal balance with descriptions and formulas are accessible in the doctor's mobile phone. A simple "angle protractor" for measuring angles before and after the operation has been adapted for medical use.

Surgimap uses such tools as "Cobb angle" and "Pelvic parameters" for measuring the parameters with its software (Fig. 2). The SpinoMeter mobile application uses the tools of "Sagittal balance. Pelvic parameters, spine parameters" for measurements (Fig. 3).

PI, PT and SS were measured in Surgimap with the tools of "Pelvic parameters"; lordoses (L1-S1) and (L4-S1) were measured with the tool "Cobb angle". The mark Cobb 1 in the figure corresponds to lordosis at L4-S1 level and the mark Cobb 2 to lordosis at L1-S1 level. SS is calculated with Surgimap as the

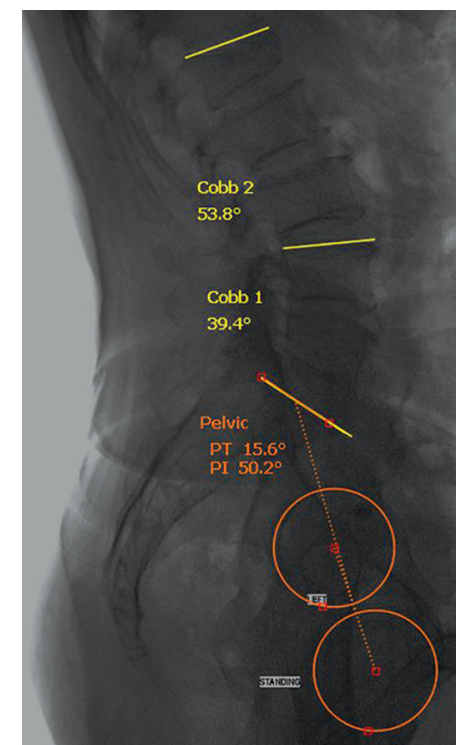

Fig. 2 Surgimap tools: Cobb angle and Pelvic parameters

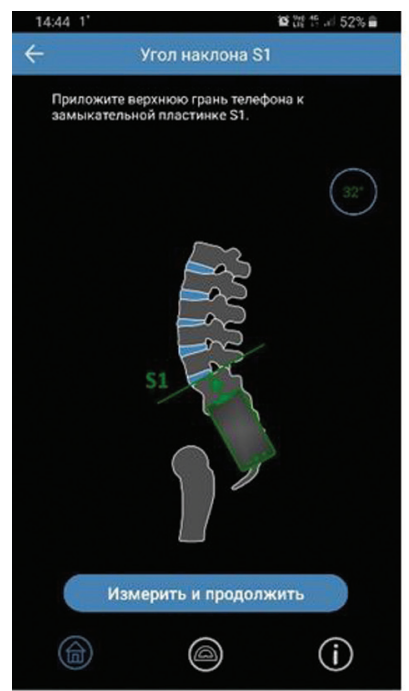

Step 1

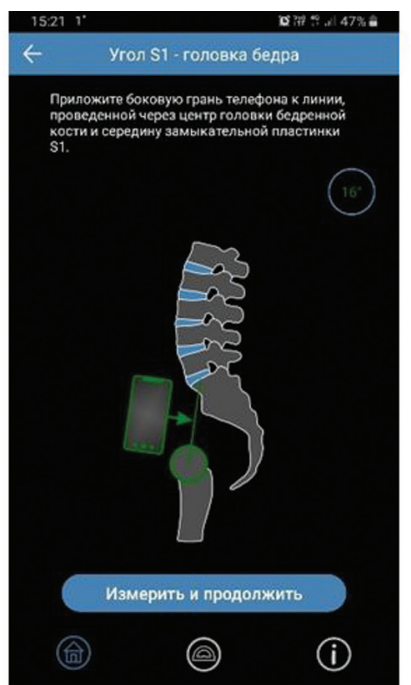

○

Step 2

difference between the values of PI and PT.

The algorithm of measurement steps with SpinoMeter for angular parameters of the sagittal balance is given in Figure 4.

Surgimap system provides the measurements of angle parameters of the sagittal balance as given in Figure 5 and SpinoMeter as shown in Figure 6.

Both Surgimap and mobile application SpinoMeter calculate the optimal values of the following sagittal balance parameters: PT, SS, L4-S1 lordosis and L1-S1 lordosis levels that are shown in the rightside columns in Figure 5 and Figure 6 and the Norm in Surgimap and Theory in the mobile application SpinoMeter.

Results of measurements (PT, SS, L4-S1 and L1S1 lordoses) were processed with MS Excel 2019 and SPSS Ver. 25.0.

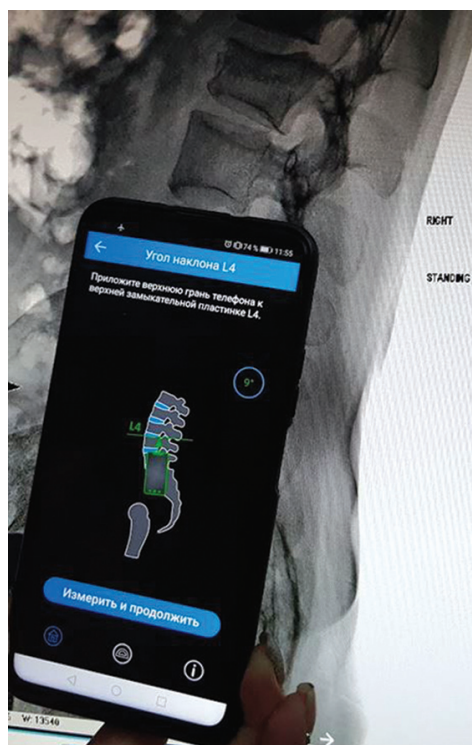

Fig. 3 Measurement of L4 angle of inclination with SpinoMeter

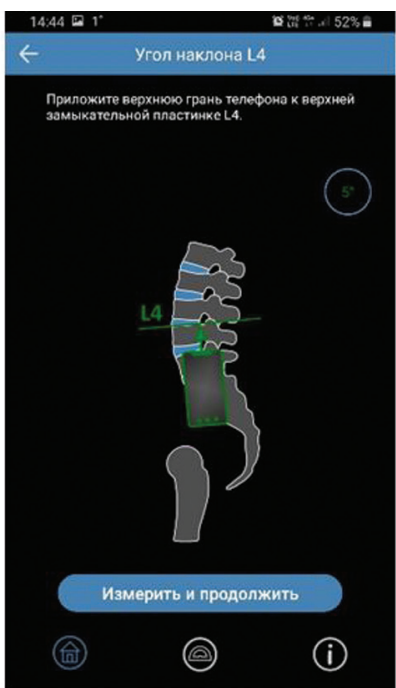

o

Step 3

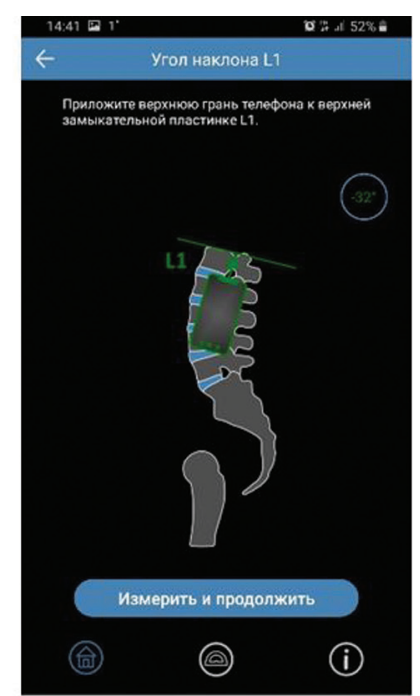

0

Step 4

Fig. 4 Steps for measuring angle parameters of the sagittal balance in the SpinoMeter mobile application: screen shots 


\begin{tabular}{|c|c|c|}
\hline Measurement Table & & B \\
\hline Measurement & Baseline & Norm \\
\hline Pelvic & & Norm \\
\hline () PT & $15.6^{\circ}$ & [7.6 to 18.4$]$ \\
\hline (2) PI & $50.2^{\circ}$ & $50.2^{\circ}$ \\
\hline () SS & $34.6^{\circ}$ & [30.6 to 43.8 ] \\
\hline (2) Cobb 1 & $39.4^{\circ}$ & \\
\hline (2) Cobb 2 & $53.8^{\circ}$ & \\
\hline
\end{tabular}

Fig. 5 Surgimap measurements of sagittal balance angles

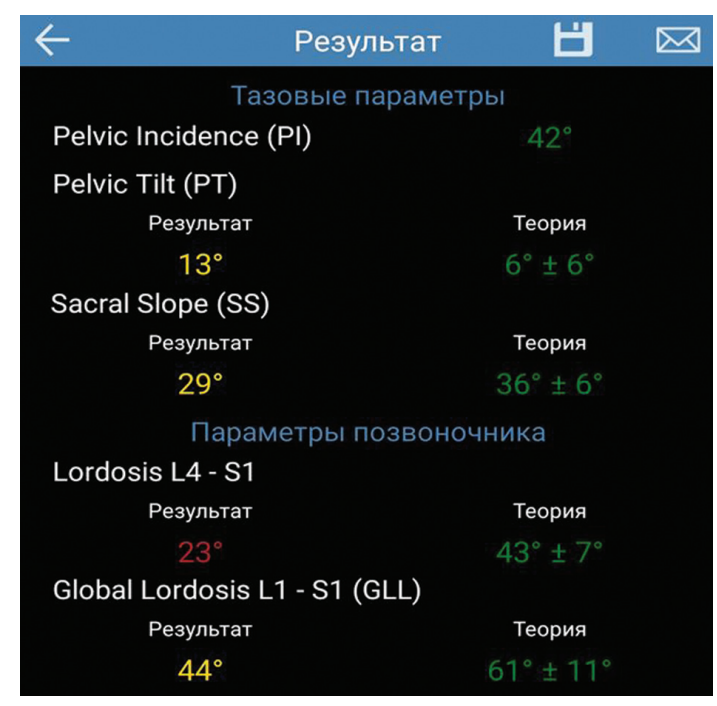

Fig. 6 SpinoMeter measurements of sagittal balance angles

\section{RESULTS}

The values of measurements of sagittal balance parameters are presented in Figures 7-11.

Next, we present the results of statistical analysis of paired samples for 52 measurements for each parameter PI, PT, SS, lordosis L1-S1, lordosis L4-S1. Descriptive statistics are given in Table 1. For normally distributed parameters, selective means are presented and in parentheses, marginal errors corresponding to the $95 \%$ confidence level. For the parameters with abnormal distribution (in the table, marked with asterisks), selected medians are presented and the ranges of the change are given in brackets.

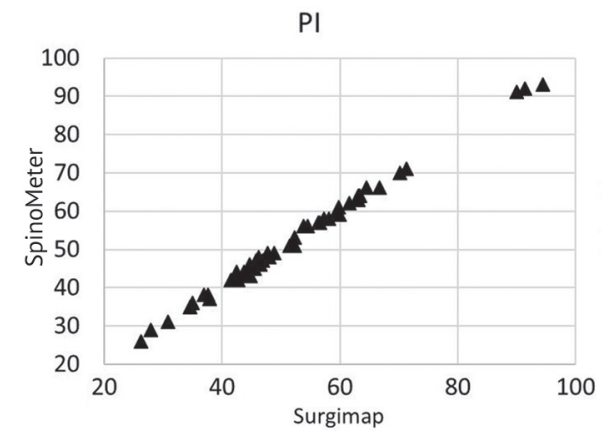

Fig. 7 Diagram of PI values measured with both software programs

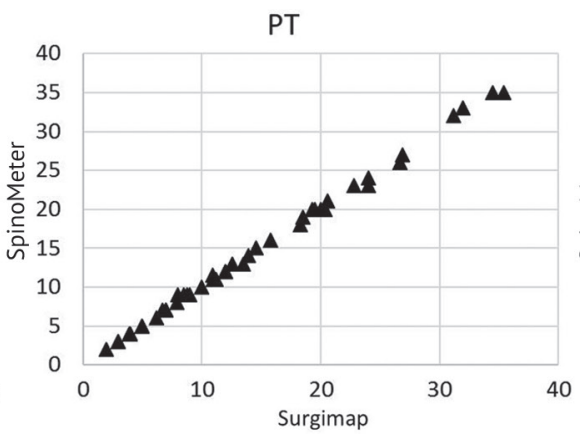

Fig. 8 Diagram of PT values measured with both software programs

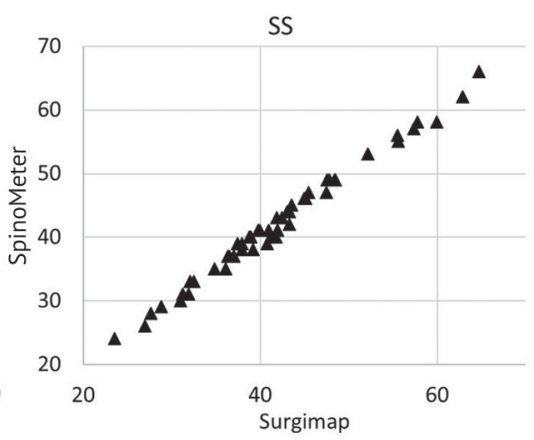

Fig. 9 Diagram of SS values measured with both software programs

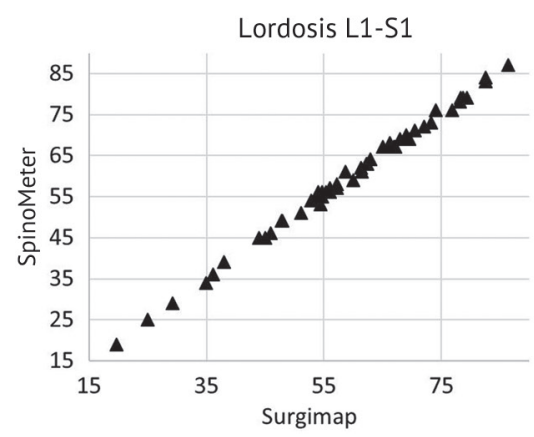

Fig. 10 Diagram of L1-S1 lordosis values measured with both software programs

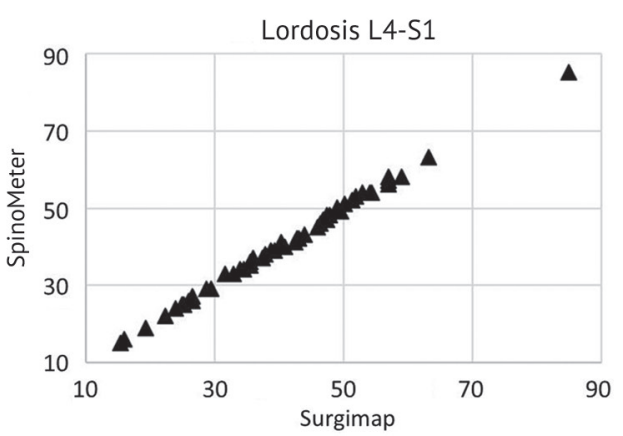

Fig. 11 Diagram of L4-S1 lordosis values measured with both software programs

Average and median values of the measured parameters of the sagittal balance

\begin{tabular}{|l|c|c|c|c|c|}
\hline \multirow{2}{*}{\multicolumn{1}{|c|}{ Programs }} & \multicolumn{5}{|c|}{ Parameters } \\
\cline { 2 - 6 } & PI & PT & SS & Lordosis L1-S1 & Lordosis L4-S1 \\
\hline Surgimap & $52.30(4.04)$ & $11.0(33.4)^{*}$ & $41.5(41.1)^{*}$ & $59.41(4.14)$ & $39.63(4.02)$ \\
\hline SpinoMeter & $52.54(4.04)$ & $11.0(33.0)^{*}$ & $42.17(2.55)$ & $59.83(4.19)$ & $40.23(3.78)$ \\
\hline
\end{tabular}


For all data, the hypothesis of the normal distribution of measurement results was tested using the Kolmogorov-Smirnov test.

To test the hypothesis about the homogeneity of measurement results for normally distributed populations, the paired Student's t-test was used. In the absence of a normal distribution, the Wilcoxon test for related samples was used.

\section{Analysis of PI measurements}

At the $5 \%$ significance level, the measurements taken by both software tools (Surgimap software, SpinoMeter mobile application) had a normal distribution. The use of the paired Student's t test at the $5 \%$ significance level confirmed the hypothesis of the uniformity of the measurement distributions.

\section{Analysis of PT measurements}

The hypothesis of a normal distribution of measurement results was rejected at a significance level of less than $0.35 \%$ for measurements made by the Surgimap software, and at a significance level of less than $0.25 \%$ for measurements made by the SpinoMetr mobile application. The Wilcoxon test for related samples at the $5 \%$ significance level confirmed the hypothesis of uniformity of measurement distributions.

\section{Analysis of SS measurements}

The hypothesis of a normal distribution of measurement results was rejected at a significance level of less than $5 \%$ for the measurements by the Surgimap but at the same significance level of $5 \%$ was confirmed for the measurements made by the SpinoMetr mobile application. The Wilcoxon's test for related samples at the $5 \%$ significance level confirmed the hypothesis of uniformity of measurement distributions.

\section{Analysis of L1-S1 lordosis measurements}

At the significance level of $5 \%$, measurements with both software tools were normally distributed. The Wilcoxon's test for related samples at a significance level of less than $0.1 \%$ rejected the hypothesis of uniformity of measurement distributions. A shift of the average value of measurements performed by the mobile application "SpinoMeter" towards decrease relative to the measurements made by the software Surgimap, not exceeding $0.7 \%$, was found.

\section{Analysis of L4-S1 lordosis measurements}

At the significance level of $5 \%$, measurements with both software tools were normally distributed. The paired Student's t-test at the 5\% significance level confirmed the hypothesis of the uniformity of the measurement distributions.

\section{DISCUSSION}

Our work is a comparative analysis of the SpinoMeter mobile application with the Surgimap desktop computer application in relation to the measurement of sagittal balance parameters on radiographs in the standing position of patients. The article also presents the distinctive features of the SpinoMeter mobile application and its functionality.

The results of our study are consistent with the work of J. Lee et al [17]. However, this study analyzed the accuracy of the SpinoMeter mobile application in comparison not with the PACS system but with the Surgimap which is the most popular preoperative planning system in Russia. Similar to J.B. Lee et al [17], our work shows that mobile applications provide high accuracy of measurements of sagittal balance parameters and in this respect are in no way inferior to applications for desktop computers. Moreover, SS measurements performed using the SpinoMeter mobile application had a normal distribution in contrast to the corresponding measurements performed by the Surgimap program. The detected shift in the measurements of L1-S1 lordosis may be due to the fact that the angle parameters were measured with the Surgimap program using different instruments ("Cobb angle" and "Pelvic parameters"). In the mobile application, all angular parameters are measured in the uniform way.

The SpinoMeter mobile application contains specific formulas and methods for calculating the optimal parameters of the sagittal balance. This is a key option for users to improve the accuracy of measurements and the validity of the results for repeated measurements in the same patient to assess the dynamics of the result [18]. This feature is absent in the Surgimap, which is a "black box" in this sense.

A significant advantage of the SpinoMeter mobile application in comparison with its analogues is the identification of patients by QR code and the ability to download measurement results into an impersonal database for further statistical analysis and their application to predict treatment results. In other words, the surgeon does not introduce the patient's data manually but only scans the patient's unique QR code containing depersonalized patient information. Thus, the erroneous data entry is excluded, and no personal data of patients is stored on the doctor's phone and is not transmitted over the Internet. Moreover, the doctor, by measuring the parameters of the sagittal balance of the patient, with his consent and the consent of the clinic, participates in the data accumulation procedure. At the same time, the measured and calculated parameters are saved in the phone, and the doctor can use them at any time and send them in text form to himself/herself or colleagues by e-mail or using the messenger installed on their phone.

In addition to the main functionality of "SpinoMeter", the function of "protractor" for measuring angles was implemented. It can be used by a doctor if it is necessary to measure any separate 
angle or for intraoperative measurements on the monitor of an image intensifier.

The options described above and the high accuracy of the SpinoMeter mobile application shown in this work makes it a unique tool for measuring and calculating the optimal parameters of the sagittal balance of the spinopelvic complex, available to every doctor.

The limitation of this study is a relatively small number of patients, and not all patients had a surgically significant sagittal imbalance.

\section{CONCLUSION}

The mobile application SpinoMeter is equivalent to Surgimap software in regard to the measurement

\section{Conflict of interests None.}

This work was supported by the Foundation for perspective studies.

\section{REFERENCES}

1. Duval-Beaupère G., Schmidt C., Cosson P. A barycentremetric study of the sagittal shape of spine and pelvis: the conditions required for an economic standing position. Ann. Biomed. Eng., 1992, vol. 20, no. 4, pp. 451-462. DOI: 10.1007/BF02368136

2. Le Huec J.C., Hasegawa K. Normative values for the spine shape parameters using 3D standing analysis from a database of 268 asymptomatic Caucasian and Japanese subjects. Eur. Spine J., 2016, vol. 25, no. 11, pp. 3630-3637. DOI: 10.1007/s00586-016-4485-5

3. Schwab F., Lafage V., Patel A., Farcy J.P. Sagittal plane considerations and the pelvis in the adult patient. Spine, 2009, vol. 34, no. 17, pp. 1828-1833. DOI: $10.1097 /$ brs.0b013e3181a13c08

4. Krutko A.V. Sagittal Balance. Harmony in Formulas. Novosibirsk, 2016, 63 p. (in Russian)

5. Makirov S.K., Yuz A.A., Jahaf M.T. Method of assessing the parameters of the sagittal spinal pelvic balance. Khirurgiia Pozvonochnika, 2015, vol. 12, no. 3, pp. 55-63. (in Russian) DOI: 10.14531/ss2015.3.55-63

6. Kudiashev A.L., Khominets V.V., Teremshonok A.V., Korostelev K.E., Nagornyi E.B., Dol A.V., Ivanov D.V., Kirillova I.V., Kossovich L.Iu. Biomechanical background for the formation of proximal junctional kyphosis after the transpedicular fixation of the lumbar spine. Rossiiskii Zhurnal Biomekhaniki, 2017, vol. 21, no. 3, pp. 313-323. (in Russian) DOI: 10.15593/RJBiomech/2017.3.05

7. Khao M., Masevnin S.V., Ptashnikov D.A., Mikhailov D.A. Assessment of the significance of sagittal balance and pathology of intervertebral discs in the development of degenerative changes in adjacent vertebral motor segments after spinal fusion. Fundamentalnye Issledovaniia, 2014, no. 10, Pt. 9, pp. 1811-1817. (in Russian) Available at: http://www.fundamental-research.ru/ru/article/view?id=36519 (accessed 21.02.2020)

8. Kim Y.L., Lenke L.G., Bridwell K.H., Kim J., Cho S.K., Cheh G., Yoon J. Proximal junctional kyphosis in adolescent idiopathic scoliosis after 3 different types of posterior segmental spinal instrumentation and fusions: incidence and risk factor analysis of 410 cases. Spine, 2007, vol. 32, no. 24, pp. 2731-2738. DOI: 10.1097/BRS.0b013e31815a7ead

9. Le Huec J.C., Charosky S., Barrey C., Rigal J., Aunoble S. Sagittal imbalance cascade for simple degenerative spine and consequences: algorithm of decision for appropriate treatment. Eur. Spine J., 2011, vol. 20, no. Suppl. 5, pp. 699-703. DOI: 10.1007/s00586-011-1938-8

10. Johnson R.D., Valore A., Villaminar A., Comisso M., Balsano M. Sagittal balance and pelvic parameters - a paradigm shift in spinal surgery. J. Clin. Neurosci., 2013, vol. 20, no. 2, pp. 191-196. DOI: 10.1016/j.jocn.2012.05.023

11. RadiAnt DICOM Viewer [Electronic resource]. Available at: https://www.radiantviewer.com/ru/ (accessed 28.02.2020).

12. Surgimap [Electronic resource]. Available at: https://www.surgimap.com/ (accessed 28.02.2020).

13. Surgimap for Android [Electronic resource]. Available at: https://play.google.com/store/apps/details?id=org.qtproject.surgimap (accessed 28.02.2020).

14. SagittalMeter Pro. [Electronic resource]. Available at: https://play.google.com/store/apps/details?id=com.sagittalmeter (accessed 28.02.2020).

15. Kotelnikov A.O., Ryabykh S.O., Burtsev A.V. «Hip-spine» syndrome: the problem from the biomechanical point of view. Genij Ortopedii, 2019, vol. 25, no. 4, pp. 541-549. (In Russian) DOI: 10.18019/1028-4427-2019-25-4-541-549

16. Akbar M., Terran J., Ames C.P., Lafage V., Schwab F. Use of Surgimap Spine in sagittal plane analysis, osteotomy planning, and correction calculation. Neurosurg. Clin. N. Am., 2013, vol. 24, no. 2, pp. 163-172. DOI: 10.1016/j.nec.2012.12.007

17. Lee J.B., Kim I.S., Lee J.J., Park J.H., Cho C.B., Yang S.H., Sung J.H., Hong J.T. Validity of a Smartphone Application (Sagittalmeter Pro) for the Measurement of Sagittal Balance Parameters. World Neurosurg., 2019, vol. 126, pp. e8-e15. DOI: 10.1016/j.wneu.2018.11.242

18. Le Huec J.C., Thompson W., Mohsinaly Y., Barrey C., Faundez A. Sagittal balance of the spine. Eur. Spine J., 2019 , vol. 28, no. 9, pp. 1889-1905. DOI: $10.1007 / \mathrm{s} 00586-019-06083-1$

Received: 13.04 .2020

\section{Information about the authors:}

1. Dmitry V. Ivanov, Ph.D. of Physico-mathematical Sciences,

Saratov State Medical University named after V.I. Razumovsky, Saratov, Russian Federation,

Email: ivanovdv@gmail.com

2. Irina V. Kirillova, Ph.D. of Physico-mathematical Sciences,

Saratov State Medical University named after V.I. Razumovsky, Saratov, Russian Federation, Email: ivkirillova@yandex.ru

3. Leonid Yu. Kossovich, Ph.D. of Physico-mathematical Sciences, Professor,

Saratov State Medical University named after V.I. Razumovsky, Saratov, Russian Federation,

Email: prezident@sgu.ru

4. Sergei V. Likhachev, M.D., Ph.D.,

Saratov State University, Saratov, Russian Federation,

Email: likha4@mail.ru

5. Asel V. Polienko,

Saratov State Medical University named after V.I. Razumovsky, Saratov, Russian Federation,

Email: st aav@mail.ru

6. Alexander V. Kharlamov, Ph.D. of Economic Sciences,

Saratov State Medical University named after V.I. Razumovsky, Saratov, Russian Federation,

Email: harlamovav@info.sgu.ru

7. Alexei E. Shulga, M.D., Ph.D.,

Saratov State University, Saratov, Russian Federation,

Email: doc.shulga@yandex.ru 